\title{
THIN FILM GaAs SOLAR CELLS ON GLASS SUBSTRATES BY EPITAXIAL LIFTOFF
}

\author{
Jack Eldredge and David Lillington \\ Spectrolab, Inc., Sylmar, CA 91342
}

X. Y. Lee, Ashish K. Verma, Charles Q. Wu, Mark Goertemiller, and Eli Yablonovitch

Dept. of Electrical Engineering, University of California at Los Angeles, Los Angeles, CA 90095

\begin{abstract}
In this work, we describe the fabrication and operating characteristics of GaAs/AlGaAs thin film solar cells using the epitaxial liftoff (ELO) technique. This technique allows the transfer of these cells onto non-absorbing glass substrates, and makes possible light-trapping operation. The enhanced performance of the lifted-off solar cell is demonstrated by means of electrical measurements under both dark and illuminated conditions.
\end{abstract}

\section{INTRODUCTION}

In recent years, III-V solar cell technology has been actively pursued for use in space applications. This is due, in large part, to the superior efficiency of these materials, compared to silicon. Attention has also been given to the fabrication of thin film cells, due to their potential for reduced weight and cost, and improved efficiency $[1,2]$. As a result, III-V cells show significant potential for terrestrial use as well. In this work, we describe the fabrication and operating characteristics of GaAs/lnGaP thin film solar cells using the epitaxial liftoff (ELO) technique [3]. This technique allows the transfer of these cells onto non-absorbing glass substrates, and makes possible light-trapping operation with enhanced performance.

\section{DEVICE FABRICATION AND LIFTOFF}

The structure used in our experiment (see Fig. 1) was grown using metal organic chemical vapor deposition. It consists of an $\mathrm{n}-\mathrm{GaAs}$ active region sandwiched between $\ln _{0.49} \mathrm{Ga}_{0.21} \mathrm{Al}_{0.3} \mathrm{P}$ and $\ln _{0.49} \mathrm{Ga}_{0.51} \mathrm{P}$ window/passivation layers, and capped by a $p^{+}-G a A s$ contact layer. The entire structure is grown on top of a $500 \AA$ thick sacrificial layer of AIAs, which is subsequently etched to release the device from its substrate. A thin GaAs layer above the AlAs serves to protect device layers during liftoff.

Devices were fabricated by first depositing Cr/AuZn/Au p-type contacts onto the sample, followed by a mesa etch down to the n-type active region. After the evaporation of AuGe/Ni/Au contacts onto the active region, the sample was annealed at $380^{\circ} \mathrm{C}$, to make the contacts ohmic. The material and junction quality were monitored after each process step using nondestructive room temperature photoluminescence [4] and open-circuit voltage $\left(V_{o c}\right)$ testing. No reduction in $V_{o c}$ was observed during the process sequence, indicating minimal parasitic leakage in the junction.

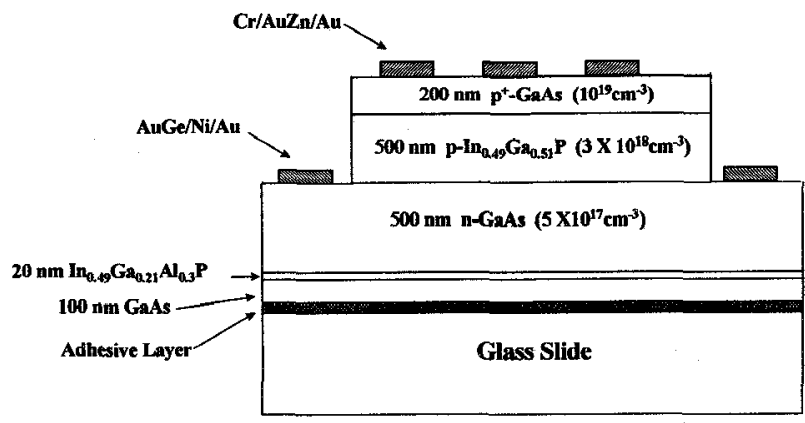

Fig. 1 Structure of the p-n GaAs solar cell.

After device fabrication, the sample was covered with black wax, as shown in Fig. 2. The thin film was lifted off of its substrate by selectively etching the AIAs layer in HF acid solution, and attached to a glass substrate with UV-curing polyurethane. The black wax was then dissolved using trichloroethylene (TCE), after which conductive brass wires were attached to the $p$ - and $n$-type contacts.

\section{ELECTRICAL MEASUREMENTS}

Electrical measurements were then performed on the lifted-off solar cell. The dark current-voltage (I-V) characteristic is shown in Fig. 3. This plot exhibits a diode factor of $n=1.93$ for voltages between $0.45 \mathrm{~V}$ and $0.9 \mathrm{~V}$, which corresponds to nonradiative recombination current. The I-V curve is virtually identical to that obtained before liftoff, indicating little material degradation during that step. It is desirable for the operating point of the cell 
A) Apply black wax:

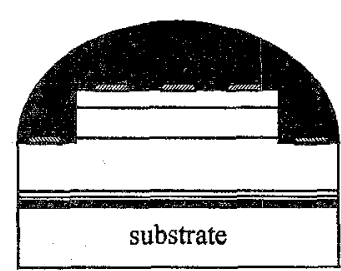

C) Attach to glass slide:

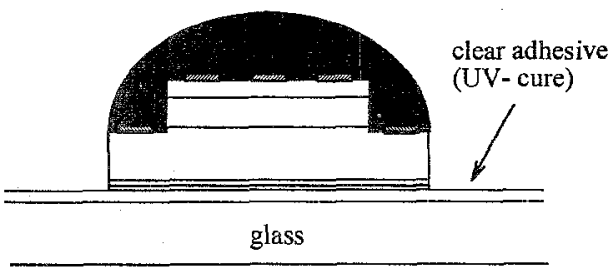

B) Liftoff in HF:

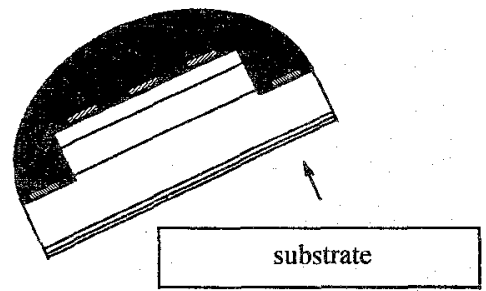

D) Dissolve wax in TCE:

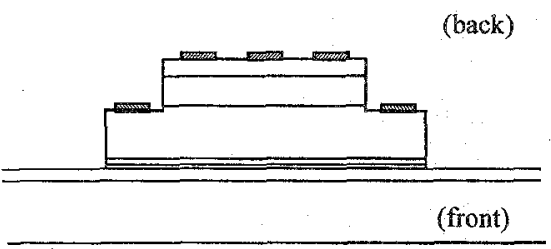

Fig. 2. Epitaxial liftoff process

to occur in the $n=1$ radiative recombination current regime, which is encountered at higher voltages. In the figure, however, this current is obscured by series resistance.

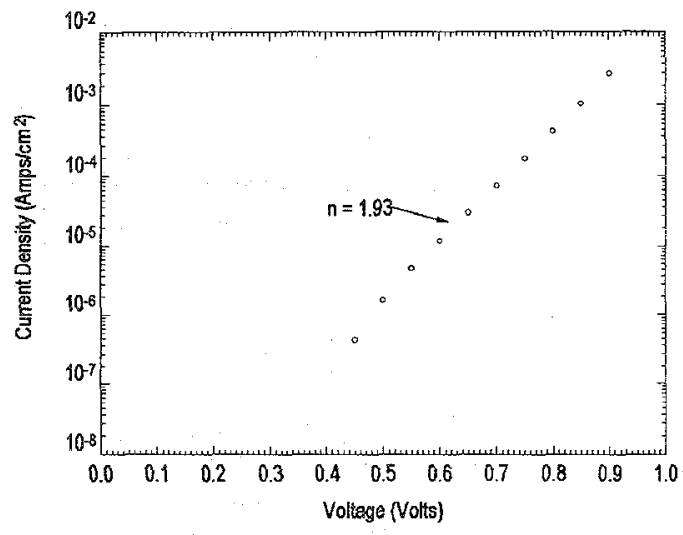

Fig. 3. Current-Voltage characteristic in the dark.

I-V curves were also obtained under an illumination condition of 1 sun (AMO). The above solar cell is bifacial, in that it can be illuminated either through the glass slide (the "front" side) or from the contact ("back") side. The result of the measurement is shown in Fig. 4. Illumination from the front yields $V_{o c}=0.995 \mathrm{~V}$ and a short-circuit current $l_{s c}$ of $30.7 \mathrm{~mA} / \mathrm{cm}^{2}$. At an operating voltage of $0.85 \mathrm{~V}$, the fill factor of this characteristic is $77 \%$ The improved performance in this configuration is due to the fact that incident light is not obstructed from the active region by metal contact pads. Moreover, reflection from the pads on the backside may enhance performance by recycling unabsorbed photons.

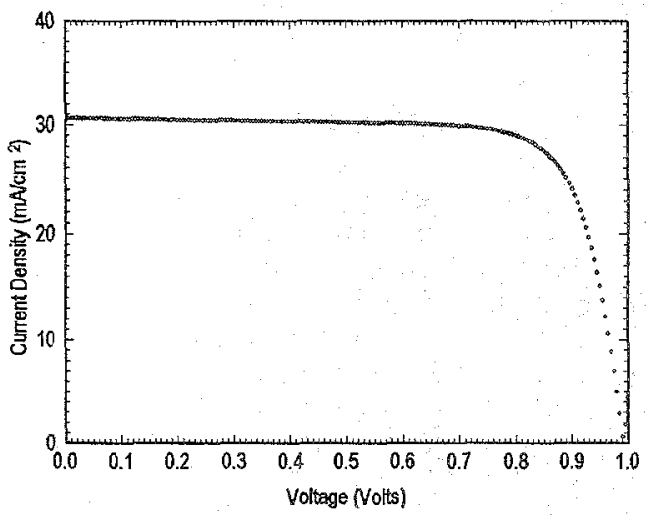

Fig. 4. Current-Voltage characteristic under 1-sun (AMO) illumination from the front side of the solar cell.

\section{DISCUSSION}

Referring to Fig. 3 , it can be seen that the operating point lies in the undesirable $n=2$ region of the 1 $\checkmark$ characteristic. This can be partially attributed to the high series resistance $(>350 \Omega$ ) in this diode, the primary source of which is the sheet resistance of the lightly doped n-type active region. Another critical factor is the bandgap of the $\ln _{0.49} \mathrm{Ga}_{0.21} \mathrm{Al}_{0.3} \mathrm{P}$ window layer. This layer was made with a low Al-content, in order to prevent its degradation during the sacrificial etch step. Consequently, a large amount of solar radiation is absorbed and lost in these layers before reaching the active region. The result is a reduction in both $V_{O c}$ and $I_{\mathrm{Sc}_{1}}$ which also tends to move the operating point into the $n=2$ regime. 
We have made a decision that future practical production of solar cells will require a InGaAlP layer with $<30 \%$ Al concentration to avoid attack by HF. Composition with $>50 \%$ are feasible in a small area device, but would be susceptible etching through pinholes in the protective GaAs layer.

\section{CONCLUSION}

In summary, we have integrated a working thin film GaAs solar cell onto a glass substrate, using the epitaxial liftoff technique. This configuration allows illumination through the non-contact side of the cell, which increases its collection area and short-circuit current. The cell exhibits $V_{o c}=0.995 \mathrm{~V}$ and $I_{s c}=30.7 \mathrm{~mA} / \mathrm{cm}^{2}$. These values are limited by series resistance and absorption in the window/passivation layers. By addressing these issues, it is expected that performance can be significantly improved.

\section{REFERENCES}

[1] G. B. Lush, M. P. Patkar, M. P. Young, M. R. Melloch, M. S. Lundstrom, S. M. Vernon, E. D. Gagnon, L. M. Geoffroy, and M. M. Sanfacon, "Thin film GaAs Solar Cells by Epitaxial Lift-off", Conf. Rec., 23rd IEEE Photovoltaic Specialists Conf., Louisville, KY, May 1993.

[2] K. Zahraman, J.-C. Guillaume, G. Nataf, B. Beaumont, M. Leroux, and P. Gibart, "High-Efficiency Al0.2 Gao.2As / Si Stacked Tandem Solar Cells using Epitaxial Lift-Off," Japanese J. of Appl. Phys., vol. 33, pp. 5807-5810, 1994.

[3] E. Yablonovitch, T. J. Gmitter, J. P. Harbison, and R. Bhatt, "Extremely Selectivity in the Lift-Off of Epitaxial GaAs Films," Appl. Phys. Lett., vol. 51, pp. 2222-2224, 1987.

[4] X.Y. Lee, C. Q. Wu, A. K. Verma, R. Ranganathan, and $E$. Yablonovitch, "Non-Destructive Testing by Absolute Room Temperature Photoluminescence Quantum Efficiency of GaAs Solar Cells," Conf. Rec., 25rd IEEE Photovoltaic Specialists Conf., Washington, D. C., May 1996. 